\section{References}

1 Manjunatha N, Kumar C, Math S, Thirthalli J. Designing and implementing an innovative digitally driven primary care psychiatry program in India. Indian J Psychiatry 2018; 60(2): 236-44.

2 Suleiman DE. Mental health disorders in Nigeria: a highly neglected disease. Ann Nigerian Med 2016; 10: 47-8.

3 Nigeria Health Care Project (NHCP). Projects. NHCP, 2021 (https://nhcp.org.uk/projects).

4 World Health Organization (WHO). mhGAP: Mental Health Gap Action Programme: Scaling Up Care for Mental, Neurological, and Substance Use Disorders. WHO, 2008 (http://www.who.int/ mental_health/mhgap_final_english.pdf).

5 Abayomi O, Adelufosi AO, Olajide A. Changing attitude to mental illness among community mental health volunteers in south-western Nigeria. Int J Soc Psychiatry 2013; 59(6): 609-12.

6 Giel R. Psychiatry in developing countries. Psychiatr Ann 1978; 8 (6): $315-20$

7 Marcus TS, Reji E, Ngcobo S. Peer-learning reviews to improve Gauteng community-oriented primary care: findings from AitaHealth ${ }^{\mathrm{TM}}$-enabled ward-based outreach teams. Afr ] Primary Health Care Fam Med 2020; 12(1): e1-10.
8 Allodola VF. The effects of educational models based on experiential learning in medical education: an internationa literature review. Tutor 2014; 14: 23-49.

9 Acharya B, Tenpa J, Basnet M, Hirachan S, Rimal P, Choudhury N, et al. Developing a scalable training model in global mental health: pilot study of a video-assisted training program for generalist clinicians in rural Nepal. Glob Ment Health 2017; 4: e8.

10 Church EA, Heath OJ, Curran VR, Bethune C, Callanan TS, Cornish PA. Rural professionals' perceptions of interprofessional continuing education in mental health. Health Soc Care Community 2010; 18(4): 433-43.

11 De Moore G, Smith K, Earle MB. From golden beaches to the heartland: reflections of NSW rural trainees. Australas Psychiatry 2006; 14(1): 72-5.

12 Berntson A, Goldner E, Leverette J, Moss P, Tapper M, Hodges B. Psychiatric training in rural and remote areas: increasing skills and building partnerships. Can J Psychiatry 2005; 50(9): 1-8.

13 Joynes V, Kerr M, Treasure-Jones T. Exploring informal workplace learning in primary healthcare for continuous professional development. Educ Prim Care 2017; 28(4): 216-22.

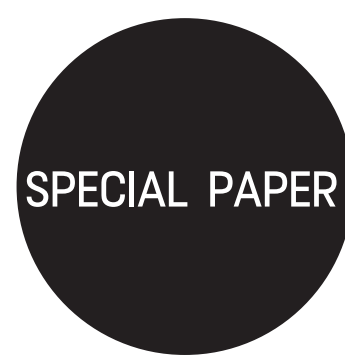

Associate Professor of Psychiatry, Government Medical College, Thiruvananthapuram, India. Email kriyalak2015@gmail.com

Keywords. Mindfulness; Indian psychotherapy; Kerala; Indian

First received 26 Sep 2020 Accepted 26 Apr 2021

doi:10.1192/bji.2021.33

c) The Author(s), 2021. Published by Cambridge University Press on behalf of the Royal College of Psychiatrists. This is an Open Access article, distributed under the terms of the Creative Commons Attribution-

NonCommercial-ShareAlike

licence (http://creativecommons. org/licenses/by-nc-sa/4.0/), which permits non-commercial re-use, distribution, and reproduction in any medium, provided the same Creative Commons licence is Crention Com is properly cited. The written permission of Cambridge University Press must be obtained for commercial re-use.

\title{
Chronicle of an Indian psychiatrist's mindfulness journey retold
}

\author{
Sivasubramoney Krishnan (i)
}

This article describes the author's discovery of mindfulness and its method and describes the bias against mindfulness even at the beginning of the 21 st century. The short essay also throws light on recent developments in mindfulness training and practice in the world and in the state of Kerala in South India.

'Sir, the Professor is on the way...' a resident ran hurriedly into the room to inform.

The meditation session in progress ceased. The projector opened its eyes onto the screen in front, tinted yellow by oldness. Slides on bipolar disorder began to roll on. The professor witnessed the class in progress with satisfaction.

The year was 2001. The venue, Mental Health Centre of the State. The attendees were about fifty nursing students who had come for their psychiatry training from various nursing colleges in the state. The class was being taken by a young psychiatrist (me!), who wanted to walk through the pathways of mental healthcare less travelled by many. The topic was mindfulness, something considered veiled, shadowy and lewd by many mental health professionals in my part of the country.

It was in 2005 that I was first exposed to the drizzle of mindfulness, during the final year of my residency programme. Before that, for the previous 8 years, I was serving as the junior-most faculty in the Department of Psychiatry of my institution (my title was Lecturer in Psychiatry, the qualification for which was an MBBS (Bachelor of Medicine and Bachelor of Surgery), the basic medical course in India). Way back in 1976 in the stress clinic of Massachusetts University, Jon Kabat Zinn had started the mindfulness revolution that in due course came to be called mindfulness-based stress reduction. ${ }^{1}$ It was generally discussed that India was the land where mindfulness originated. Yet there were only a few centres in India (unknown to me and most other people) to impart training on this. Curious about and fascinated by the meditations of Jon Kabat Zinn, Emeritus Professor of Medicine at the University of Massachusetts Medical School, and the world of his classic masterpiece Full Catastrophe Living, ${ }^{2}$ I journeyed into the world engulfed by a little book - The Miracle of Mindfulness - penned by Thich Nhat Hanh, a Vietnamese monk. ${ }^{3}$ By the time I started to lay my initial steps, mindfulness, the Eastern contemplative practice rooted in Buddhism, had been reasonably well integrated into the fields of medicine and behavioural health, grounded in Western science. ${ }^{4}$ Still, I was reading about and attempting to practise a method that was equated with having schizophrenia among professionals in my part of the country.

Jon Kabat Zinn defined mindfulness as the awareness cultivated by non-judgemental, open, 
curious attention to the unfolding of momentto-moment experience. ${ }^{2}$ Another book of his, Wherever You Go, There You Are, is an easy-to-read map of the many ways in which mindfulness can be inserted into our busy Western lives. ${ }^{5}$ This gradually paved the way for reading on other stalwarts in the field of mindfulness, such as Sam Harris, Saki Santorelli, Sara Lazar and Daniel Goleman. To quote Sam Harris,

'There is nothing passive about mindfulness. One might even say that it expresses a specific kind of passion-a passion for discerning what is subjectively real in every moment. It is a mode of cognition that is, above all, undistracted, accepting, and (ultimately) nonconceptual. Being mindful is not a matter of thinking more clearly about experience; it is the act of experiencing more clearly, including the arising of thoughts themselves. Mindfulness is a vivid awareness of whatever is appearing in one's mind or body-thoughts, sensations, moodswithout grasping at the pleasant or recoiling from the unpleasant'.

Reading about Saki Santorelli and his efforts in training medical professionals in mindfulness was really an enchanting treatise. ${ }^{7}$ His book Heal Thyself paved the way for the young psychiatrist's dream to grow wings. ${ }^{8}$ Sara Lazar's study on the enhanced cortical thickness due to mindfulness meditation was yet another step in his journey. ${ }^{9}$ The slogan 'nothing comes, nothing stays and nothing goes', describing the different stages of mindfulness meditation practice in Daniel Goleman's book, became a quote to carry in mind and body. ${ }^{10}$ The pathway ultimately ended in the trio of Mark Williams, John Teasdale and Zindel Segal, who had formulated the concepts of mindfulness-based cognitive therapy for prevention for depressive relapse. ${ }^{11}$

Most of the people around had no idea other than that mindfulness is a Buddhist meditation technique. Books, journals or training centres on the subject were unheard of in our part of the country. Many opined that it was an intellectual fraud or an escapist method or a measure of one's surrendering to life's moments. Yet to my awe I saw that the experiential concept of mindfulness was equally acceptable to Western culture, which valued knowledge and material rewards, and to our own Eastern culture, which emphasised philosophies that attempted to explore the meaning of life. Readings on the yesteryears of mindfulness made me aware that mindfulness is a culture-sensitive practice which is not confined to any specific religion or culture. Different cultures had their own ways of enhancing awareness through compassionate attention, i.e. mindfulness.

\section{Bringing mindfulness into clinical practice in India}

In 2009, I organised the first mindfulness session with a patient with depression, his wife and a staff nurse of the hospital. The four of us sat in a large hall and meditated mindfully for the first the time in the history of our institution. In the same year, on behalf of the Holistic and Psychosomatic Clinic of the Department of Psychiatry at the Government Medical College, Thiruvananthapuram, the first mindful life management (MLM) stress management workshop was organised. Lasting $150 \mathrm{~min}$, this gradually paved the way for half-day and full-day workshops. To date, more than 500 workshops have been conducted.

In 2016, we thought of a psychotherapy module for use by mental health professionals for specific psychiatric disorders. Drawing on mindfulness-based stress reduction (MBSR), ${ }^{2}$ mindfulness-based cognitive therapy (MBCT), ${ }^{11}$ mindfulness-integrated cognitive-behavioural therapy $(\mathrm{MiCBT}),{ }^{12}$ acceptance and commitment therapy (ACT) $)^{13}$ and our own little programme of mindful life management (MLM) we formulated mindfulness-unified cognitive-behavioural therapy (MUCBT). We organised the first statelevel training programme for about 20 mental health professionals, including psychiatrists, psychologists and counsellors. In 2018, we organised the first national-level workshop on MUCBT for 20 selected mental health professionals from all over India. To my good fortune, I was able to attend a teachers' training workshop organised by Oxford Mindfulness Centre (OMC) in 2017, with Dr Wilhem Kuyken, the then Director of OMC, as the chief trainer. Kuyken and his studies on depression and mindfulness were an inspiring experience to cherish for a long time. And over the past 3 years, two national workshops and a 100-session webinar on different topics related to mindfulness have materialised. The webinar is in progress and covers topics ranging from what mindfulness is to its neurobiology and future. Our state today is the first state in India to have introduced mindfulness for a state police force, producing nearly 25 trainers. We have also organised mindfulness training for mental health professionals in different parts of India, medical students, oncologists and teachers. The programme today is offered free of cost from the Government Medical College, Thiruvananthapuram, in South Kerala.

\section{How far mindfulness has come}

Learned as a method of stress management for my own self and to mitigate the suffering of the patients whom I care for, over the years, I discovered that mindfulness is an easy method that could be incorporated into the moments of life. Today, mindfulness is a word equally near and dear to the homes of millions across the world and to the citadels of scientists. ${ }^{14}$ Both as a household tool for wellness and as a management strategy for mental and physical disorders, mindfulness finds its place in healthcare. ${ }^{15}$ It also has a major role in the areas of education, ${ }^{16}$ management and the workplace. ${ }^{17}$

Mindfulness helped me to attempt listening better, attempt to be present with less distraction, 
attempt to accept my annoyance over the little things, face challenges by looking at them from a new perspective, attempt to communicate better, understand the array of emotional fluctuations and to feel a deeper sense of connection and gratitude. Perhaps the best part of mindfulness training was the feeling of a deeper sense of connection and gratitude. Some nights are more difficult than others. Mindfulness helps me to surf through the troughs and gutters of life with compassionate awareness.

Today, after nearly a decade and half has glided under the bridge of time, mindfulness has entered the mainstream medical arena with dreams of a better tomorrow. In the area of mental healthcare, mindfulness-based interventions (MBIs) find application in the treatment of a large number of psychiatric disorders. There is evidence in support of this in stress management, ${ }^{18,19}$ pain management, ${ }^{20,21}$ depression, ${ }^{22-24}$ anxiety, ${ }^{24-26}$ borderline personality disorder, ${ }^{27}$ addiction management, ${ }^{28}$ relapse prevention ${ }^{29}$ and the like. During periods of severe crisis, MBIs have been found to be of benefit to the community. ${ }^{26}$ MBIs benefit not only the patient population, but also healthcare staff, including physicians. ${ }^{30,31}$

On the same road, there are parallel attempts to trivialise mindfulness as a quick fix to generate happiness, health and a balanced life. The cold war, though muffled now, continues on its own way. Is mindfulness a hope or hype? - the question is openly discussed now.

Mindfulness, I feel, is the practice of experiencing each moment of our day-to-day life as it happens. As Jon Kabat Zinn suggested, the introduction of mindfulness into the mental health field definitely has a potential to change the face of mental healthcare.

\section{Funding}

This research received no specific grant from any funding agency, commercial or not-for-profit sectors.

\section{Declaration of interest}

None.

\section{References}

1 Kabat-Zinn J. Some reflections on the origins of MBSR, skillful means, and the trouble with maps. Contemp Buddhism 2011; 12: $281-306$

2 Kabat-Zinn J. Full Catastrophe Living: Using the Wisdom of Your Body and Mind to Face Stress, Pain, and IIIness. Bantam Books, 2013.

3 Thich NH. The Miracle of Mindfulness: A Manual on Meditation. Beacon Press, 1987.

4 Polanski PJ. Mindfulness in context.Counsell Spirituality 2015; 34 (2): 15-28.

5 Kabat-Zinn J. Wherever You Go, There You Are: Mindfulness Meditation in Everyday Life. Hyperion, 1994.

6 Harris S. Waking up: A Guide to Spirituality without Religion. Simon \& Schuster, 2014.

7 Santorelli SF. Saki F. Santorelli. Omega, 2012 (https://www.eomega. org/workshops/teachers/saki-f-santorelli [cited 27 Aug 2020]).
8 Santorelli S. Heal Thy Self: Lessons on Mindfulness in Medicine. Belltower, 2000.

9 Lazar SW, Kerr CE, Wasserman RH, Gray JR, Greve DN, Treadway MT, et al. Meditation experience is associated with increased cortical thickness. Neuroreport 2005; 16 : 1893-7.

10 Goleman D, Davidson RJ. Altered Traits: Science Reveals How Meditation Changes Your Mind, Brain, and Body. Avery Publishing Group, 2017.

11 Segal Z, Williams M, Teasdale J. Mindfulness-Based Cognitive Therapy for Depression. Guilford Press, 2013.

12 Cayoun BA. Mindfulness-Integrated CBT for Well-Being and Personal Growth. Wiley Blackwell, 2015.

13 Hayes SC, Strosahl KD, Wilson KG. Acceptance and Commitment Therapy: The Process and Practice of Mindful Change (2nd edn). Guilford Press, 2012.

14 Sacchet M. Mindfulness is more than a buzzword: a look behind the movement. Forbes 2015; 29 Sep (https://www.forbes.com/ sites/payout/2017/09/29/mindfulness-is-more-than-a-buzzworda-look-at-the-neuroscience-behind-the-movement/ \#13bef3e8372f [cited 26 Aug 2020 ]).

15 Tlalka S. Meditation is the fastest growing health trend in America. Mindful 2018; 11 Dec (https://www.mindful.org/ meditation-is-the-fastest-growing-health-trend-in-america/ [cited 26 Aug 2020]).

16 Batra SV. The importance of mindfulness in education mindful or mind fooled. Businessworld 2018; 28 Jul (http://www. businessworld.in/article/The-Importance-Of-Mindfulness-InEducation-Mindful-Or-Mind-fooled/28-07-2018-155538/ [cited 26 Aug 2020]).

17 Craig H. Mindfulness at work: using mindful leadership in the workplace. PositivePsychology.com 2019 (https://positive psychology.com/mindfulness-at-work/ [cited 26 Aug 2020]).

18 Sharma M, Rush SE. Mindfulness-based stress reduction as a stress management intervention for healthy individuals: a systematic review. J Evid Based Complement Altern Med 2014; 19: 271-86.

19 Chiesa A, Serretti A. Mindfulness-based stress reduction for stress management in healthy people: a review and meta-analysis. J Altern Complement Med 2009; 15: 593-600.

20 Majeed MH, Ali AA, Sudak DM. Mindfulness-based interventions for chronic pain: evidence and applications. Asian J Psychiatr 2018; 32: 79-83.

21 Skaer T. Research findings using mindfulness-based interventions for chronic pain. Pain Stud Treatment 2015; 3: $38-45$.

22 van der Velden AM, Kuyken W, Wattar U, Crane C, Pallesen KJ, Dahlgaard J, et al. A systematic review of mechanisms of change in mindfulness-based cognitive therapy in the treatment of recurrent major depressive disorder. Clin Psychol Rev 2015; 37 26-39.

23 Williams J, Kuyken W. Mindfulness-based cognitive therapy: a promising new approach to preventing depressive relapse. $\mathrm{Br}$ ] Psychiatry 2012; 200: 359-360.

24 Hofmann SG, Sawyer AT, Witt AA, Oh D. The effect of mindfulness-based therapy on anxiety and depression: a meta-analytic review. J Consult Clin Psychol 2010; 78: 169-83.

25 Rodrigues MF, Nardi AE, Levitan M, Rodrigues MF, Nardi AE, Levitan M. Mindfulness in mood and anxiety disorders: a review of the literature. Trends Psychiatry Psychother 2017; 39: 207-15.

26 Behan C. The benefits of meditation and mindfulness practices during times of crisis such as COVID-19. Ir J Psychol Med 2020; 37: $256-8$. 
27 Carmona i Farrés C, Elices M, Soler J, Domínguez-Clavé E, Pomarol-Clotet E, Salvador R, et al. Effects of mindfulness training on borderline personality disorder: impulsivity versus emotional dysregulation. Mindfulness 2019; 10: 1243-54.

28 Garland EL, Howard MO. Mindfulness-based treatment of addiction: current state of the field and envisioning the next wave of research. Addict Sci Clin Pract 2018; 13: 14. $\mathrm{M}$, et al. Mindfulness-based relapse prevention for substance use disorders: a systematic review and meta-analysis. J Addict Med 2017; 11: 386-96.

30 Burton A, Burgess C, Dean S, Koutsopoulou GZ, Hugh-Jones S. How effective are mindfulness-based interventions for reducing stress among healthcare professionals? A systematic review and meta-analysis. Stress Health 2017; 33: 3-13.

31 Lomas T, Medina JC, Ivtzan I, Rupprecht S, Eiroa-Orosa FJ. A systematic review and meta-analysis of the impact of mindfulness-based interventions on the well-being of healthcare professionals. Mindfulness 2019; 10: 1193-216.

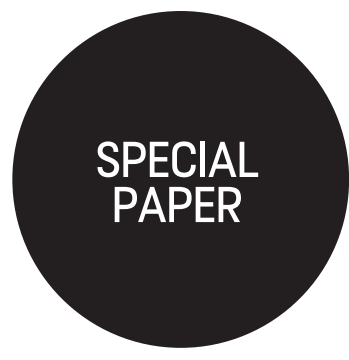

\title{
Recognising and healing emotional wounds of child labourers: call to action based on the evidence and stakeholder views from India and Nepal
}

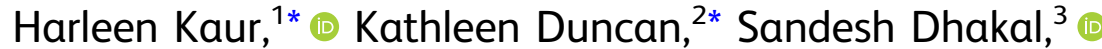 \\ Narayan Sharma, ${ }^{4}$ Shanta Niraula, ${ }^{5}$ Rakesh Pandey, ${ }^{6}$ Veena Kumari, ${ }^{7}$ \\ Jennifer Y. F. Lau ${ }^{8} \odot$ and Tushar Singh ${ }^{9} \odot$
}

${ }^{1} \mathrm{MSc}$, Research Scholar Department of Psychology, Banaras Hindu University, Varanasi, India

${ }^{2}$ Student, Division of Psychology \& Language Sciences, University College, London, UK

${ }^{3}$ PhD, Lecturer, Central Department of Psychology, Tribhuvan University, Kathmandu, Nepal

${ }^{4} \mathrm{PhD}$, Lecturer and Head of the Department, Department of Philosophy and Psychology, Tri-Chandra Campus, Ghantaghar, Kathmandu Nepal

${ }^{5} \mathrm{PhD}$, Former Head, Central Department of Psychology, Tribhuwan Univers

${ }^{6} \mathrm{PhD}$, Professor, Department of Psychology, Banaras Hindu University, Varanasi, India

${ }^{7} \mathrm{PhD}$, Professor, Centre for Cognitive Neuroscience, College of Health, Medicine and Life Sciences, Brunel University London, UK

${ }^{8} \mathrm{PhD}$, Professor of Youth Resilience, Youth Resilience Research Unit, Wolfson Institute of Population Health, Queen Mary University of London, UK

${ }^{9}$ DPhil, Assistant Professor, Department of Psychology, Banaras Hindu University,

anasi, India.

Email: tusharsinghalld@gmail.com

*Authors contributed equally.

Keywords. Child labourers; mental health; mental health interven

tions; care providers;

psychoeducation.

First received 10 Feb 2021 Final revision 23 Jun 2021 Accepted 21 Jul 2021
Child labourers are at risk of poorer mental health and once rescued require urgent mental health interventions to ameliorate the long-term impact. In our review, only two published scientific studies evaluated custommade interventions; other programmes were obtained from non-governmental organisations (NGOs), which need rigorous trial evaluation. We also sought the viewpoints of stakeholders working directly with rescued young people, as well as consulting young people with lived experiences of child labour. We propose that psychoeducational interventions aimed at employees working directly with young people could represent a fruitful approach for lowand middle-income countries in the AsiaPacific region but also more generally.

Child labour, a pressing human rights issue, is prevalent in many Asian low- and middle-income countries (LMICs), with a report released in 2017 by the International Labour Organization (ILO) citing around 1 in 14 young people employed illegally in the Asia-Pacific region. ${ }^{1}$ Child labour is defined by the ILO on the basis of its harmful consequences, most notably, its interference with schooling. Indeed, data show the impact of child labour on school attendance and educational attainment, ${ }^{2}$ which in turn contributes to the intergenerational transmission of poverty within families $^{3}$ and reduced accumulation of human capital for economies. Eliminating child labour is therefore an increasing focus of intergovernmental and non-governmental organisations (NGOs) but until these efforts completely succeed, the provision of support to young people currently working illegally or those who have been rescued is an urgent practical task. Indeed, child labour involves work that is 'mentally, physically, socially or morally dangerous and harmful to children'. Recently, we also uncovered widespread experience of maltreatment among rescued adolescent labourers in India and Nepal. Using the Juvenile Victimization Questionnaire (JVQ), ${ }^{5}$ a large proportion of each sample reported exposure to childhood abuse and/or neglect $(83.36 \%$ in the India sample, $72 \%$ in the Nepal sample). The most common experiences were physical abuse (reported by $46.6-72.7 \%)$ and emotional abuse ((40.8-47.7\%). ${ }^{6,7}$ These experiences were associated with poor mental health, particularly symptoms of affective disorders, ${ }^{6,7}$ consistent with other published scientific studies. ${ }^{8-13}$ Speaking more directly to these points, our research team also consulted with six care-home employees (who had between 2 and 11 years of experience working directly with young labourers in India and Nepal) both with and without social work or counselling backgrounds. The sample comprised stakeholder consultants comprised both front-line care staff and those in management positions, including the founder of an NGO. These consultations also firmly indicated that mental health interventions were an urgent priority. One social worker in a care-home in India said:

'Mental health is utmost important, as I told you earlier also, the capacity of a child to think, to understand someone or some situation has completely vanished [...] They have printed in their thinking that they are born to be exploited, they can only experience the emotions of fear and sadness, 